\title{
Review of Hybridizations of Kalman Filters with Fuzzy and Neural Computing for Mobile Robot Navigation
}

\author{
Manuel Graña, Iván Villaverde, Jose Manuel López Guede, Borja Fernández* \\ Grupo de Inteligencia Computacional, UPV/EHU, \\ www.ehu.es/ccwintco
}

\begin{abstract}
Kalman Filters (KF) are at the root of many computational solutions for autonomous systems navigation problems, besides other application domains. The basic linear formulation has been extended in several ways to cope with non-linar dynamic environments. One of the latest trend is to introduce other Computational Intelligence (CI) tools, such as Fuzzy Systems or Artificial Neural Networks inside its computational loop, in order to obtain learning and advanced adaptive properties. This paper offers a short review of current approaches.
\end{abstract}

\section{Introduction}

Navigation, defined as the the process of determining and maintaining a course or trajectory to a goal location[8], has been considered the most basic and key problem in mobile robotics since the beginning of the research on that area. Opposite to a "wandering" behavior of the robot, in which it simply "moves around" by just avoiding obstacles in its path, navigation implies a knowledge of the structure of its environment and the ability to self-locate itself in it and in respect to its goal. That first condition requires that the robot has some a priori information about its surroundings. Autonomy increases inversely withe a priori information needed by the system to navigate in its environment. Maps[23] are the world representation used to guide navigation. The most autonomous form of navigation is Simultaneous Localization and Mapping (SLAM) $6,1,7]$, in which the robot builds a map while localizing itself in it, and uses this position estimation to integrate new measurements from the environment into the previous map. This basic dynamic models in SLAM are probabilistic, and the computational solutions are stochastic ones such as Expectation Maximization algorithms, Monte Carlo particle filters and Kalman Filters (KF). The latter were developed in the early 60's by R. E. Kalman and R. S. Bucy[11, 24]. The origin of the use of Kalman Filters in mobile robotics can be traced back to the 80 's, when the actual formulation was introduced by Smith, Cheeseman and Self $[22,21]$.

\footnotetext{
* The Spanish Ministerio de Educacion y Ciencia supports this work through grant DPI2006-15346-C03-03
} 
Because the $\mathrm{KF}$ is in essence a linear method, the classical extensions found in the literature to cope with non-linear phenomena are linearization strategies. In this paper we explore the diverse hybridizations of the KF with of Computational Intelligence (CI) tools, which follow an intrinsic non linear modelling approach. These hybridizations may help to improve the accuracy and tracking power of the KF. This survey is intended to help opening avenues for research $n$ Hybrid Systems and their applications in mobile robotics. We have found that the main KF hybridizations are: (1) using KF as an estimation algorithm to train computational systems (Artificial Neural Networks, Fuzzy Systems), instead of the simple gradient descent algorithms, (2) using CI tools to model the KF elements more realistically, (3) mixing EKF with other (fuzzy) representations.

The structure of the paper is as follows: We give a short review of the Kalman filters $(\mathrm{KF})$ and their variants that have found application in mobile robotics in section 2 . In section 3 we present the approaches to hybrid KF. Finally, section 4 gives final conclusions and comments.

\section{Kalman Filters and non-linear variants}

In a typical mobile robot SLAM navigation, the robot moves around taking measurements of its environment trying to self-localize in the world, and uses them also to build a map to refer this localization. Uncertainties in the measurement come from the sensor noise, and from the inaccurate realization of the commanded actions (i.e. wheel skid). Formally, at each time instant $k$ the robot would be at state $x_{k} \in \Re^{n}$, and its motion is assumed to follow a given model $x_{k}=f\left(x_{k-1}, u_{k-1}, w_{k-1}\right)$, which becomes $x_{k}=A_{k} x_{k-1}+B_{k} u_{k}+w_{k}$ in the linear case, where $w_{k-1}$ is the motion noise, and $u_{k-1}$ the motion command issued to the system at time $k-1$. At each time instant the robot makes some environment measurements $z_{k}$ that can be assumed to follow a model $z_{k}=h\left(x_{k}, v_{k}\right)$ of the system state $x_{k}$ and observation noise $v_{k}$, which becomes $z_{k}=H_{k} x_{k}+v_{k}$ in the linear case.

The KF is a recursive method to estimate the state of a system. The knowledge of state of the system at time $k$ will be represented by its estimation $\hat{x}_{k}$ and its related error covariance matrix $P_{k}$. This error covariance is a measure of the accuracy of the state estimation, defined as $P_{k}=E\left[e_{k} e_{k}^{t}\right]$, being $e_{k}=x_{k}-\hat{x}_{k}$ the estimation error. State estimation is performed by means of a two step prediction-correction feedback process. In the first step, the Prediction step, an a priori estimation of the state $\left(\hat{x}_{k}^{-}\right.$and $\left.P_{k}^{-}\right)$is done. Then, measurements of the environment are taken into account in a second step, the Correction step, to compute the a posteriori estimation $\left(\hat{x}_{k}^{+}\right.$and $\left.P_{k}^{+}\right)$, which becomes the sytem estimation $\left(\hat{x}_{k+1}\right.$ and $\left.P_{k+1}\right)$ at time $k+1$. This recursive computation schema is the core of the $\mathrm{KF}$, and all their variants.

The Discrete Kalman Filter (DKF) is the classical formulation of the linear $\mathrm{KF}$ for linear problems with known noise covariance matrices, the well know Prediction-Correction equations are as follows:

$$
\hat{x}_{k}^{-}=A \hat{x}_{k-1}+B u_{k-1} ; P_{k}^{-}=A P_{k-1} A^{T}+Q,
$$




$$
\hat{x}_{k}^{+}=\hat{x}_{k}^{-}+K_{k}\left(z_{k}-H \hat{x}_{k}^{-}\right) ; P_{k}^{+}=\left(I-K_{k} H\right) P_{k}^{-},
$$

where $K_{k}$ is known as the Kalman Gain, and is a factor that relates the uncertainty between the state estimation and the residual prediction error.

The Extended Kalman Filter (EKF) is an improvement of the DKF to deal with non-linear problems. Assumed that the non-linear model is known, the state is predicted according to its model, and aPredictive step and the performed observation follows the bare application of this model:

$$
\hat{x}_{k}^{-}=f\left(\hat{x}_{k-1}, u_{k-1}, 0\right) ; \hat{z}_{k}^{-}=h\left(\hat{x}_{k}^{-}, 0\right) .
$$

A linear approximation of the non-linear model is performed at the point $\left(\hat{x}_{k}^{-}, \hat{z}_{k}^{-}\right)$:

$$
x_{k} \approx \hat{x}_{k}^{-}+A\left(x_{k-1}-\hat{x}_{k-1}\right)+W w_{k-1} ; z_{k} \approx \hat{z}_{k}^{-}+H\left(x_{k}-\hat{x}_{k}^{-}\right)+V v_{k},
$$

where $A$ and $W$ are the Jacobian matrices of $f$ relative to $x$ and $w$, respectively, and $H$ and $V$ are the Jacobian matrices of $h$ relative to $x$ and $v$, respectively. From this point the EKF is equal to a DKF, it applies the coorresponding equations adapted from 1 and 2. EKF is not an optimal estimator (as the DKF was) and it is very sensitive to wrong initial condition estimation and bad process models. The way it linearizes the functions it is also source of inefficiency. As second and higher terms of the Taylor series are discarded, if those terms are not negligible, it introduces error and bias in the transformation, as the local linearity assumption is broken. Also, the derivation of the Jacobian matrices is not usually trivial and can be very difficult in some applications.

The Unscented Kalman Filter (UKF) [9] replaces the linear approximation of the EKF with the unscented transformation. The aim of this transformation is to calculate the statistics of a random variable which undergoes a non-linear transformation. The basic idea is that is easier to approximate a probability distribution than an arbitrary non-linear function, and it does so by applying the non-linear function to a set of points $\left\{\chi_{i}\right\}$ sampled deterministically from a hypothetical population of points with mean $\bar{x}$ and spatial covariance $P_{x}$ and calculating the mean and covariance of the transformed points. The non-linear dynamic system model is applied to these points $\gamma_{i}=f\left[\chi_{i}\right]$ and first and second order statistics are computed (applying convenient weights $\left\{W_{i}\right\}$ ):

$$
\bar{y}=\sum_{i=0}^{2 n} W_{i}^{(m)} \gamma_{i} ; P_{y}=\sum_{i=0}^{2 n} W_{i}^{(c)}\left(\gamma_{i}-\bar{y}\right)\left(\gamma_{i}-\bar{y}\right)^{T} .
$$

The UKF uses these estimations as the a priori estimation computed at the Prediction step, $\hat{x}_{k}^{-}=\bar{y}$ and $P_{k}^{-}=P_{y}$ as computed in 5 . The system observation prediction $\hat{z}_{k}^{-}$and its covariance matrix $P_{z_{k}}$ is computed in an analogous way. Finally, the correction step is computed:

$$
\hat{x}_{k}=\hat{x}_{k}^{-}+K_{k}\left(z_{k}-\hat{z}_{k}^{-}\right) ; P_{k}=P_{k}^{-}-K_{k} P_{z_{k}} K_{k}^{T},
$$

where the gain is computed as $K_{k}=P_{x_{z} y_{z}} P_{z_{k}}^{-1}$. 
Other variants proposed in the literature try to cope with non-stationary processes by estimating the noise covariance matrix at each time step, or work with agregates instead of the original variables to reduce the dimensionality of the state vector [12], or merge KF with Monte Carlo techniques [15].

\section{Hybrized approaches}

We will consider three kind of hybridizations found in the literature: The use of $\mathrm{KF}$ as estimation (learning, tuning) algorithm, the enhancement of KF elements with nonlinear modeling abilities, and the mixture of representations. Although the first one is not greatly relevant to mobile robotics navigation, we discuss it for the sake of completeness.

\subsection{KF as an estimation algorithm}

The KF can be viewed as a parameter estimation algorithm with the added ability to take into account the uncertainty of the estimation to provide an adaptive gain in the learning steps. It can be, thus, used as the training algorithm for the CI leaning approaches, such as Artificial Neural Networks or Fuzzy Systems. Following this philosophy, the EKF has been applied as the basic learning algorithm for the estimation of Radial Basis Functions (RBF) parameters [14, 20] inside a growing architecture. The Node Decoupled learning applies an EKF to each network node independently, the system state corresponds to the RBF weights, both hidden units mean and variance and the hidden to output weights, the observation being the desired output. The Kalman gain is computed on an estimation of the network prediction error covariance, assuming a constant noise distribution. EKF improves over gradient descent because it introduces an adaptive learning gain (the Kalman gain) depending on the uncertainty of the network prediction, thus ensuring smooth convergence. The EKF has been also applied to tuning the membership functions in Mamdani type of fuzzy systems with correlation inference [19]. There the estimation of the optimal membership parameters is found equivalent to nonlinear dynamic system identification problem, solved applying the EKF.

\subsection{Non-Linear Enhancement of KF}

There have been some attemps to embedd CI algorithms to relax the strong assumptions of the KF and its extensions (EKF, UKF). Superseding the linear dynamical and observation model, relaxing the constraints on the assumed noise distribution or the computation of the Kalman gain are some of the KF algorithm elements that have beed influenced by CI techniques.

Because EKF relies heavily on the assumption of white noise, it can ben enhanced by introducing a noise covariance estimation algorithm that can cope with coloured noise or systematic error bias. The work in [4] uses Artificial Neural Networks to model the noise in the motion and sensing of a mobile robot, 
showing improvements over the accuracy of positioning. The main handicap of their approach is that the ANN training must be performed off-line, so the environment has to be well defined, as well as the robot characteristics. For SLAM applications it is desirable that the noise modeling is done on-line with the state updating and control. This endeavor is taken in [3] where they apply a Neuro-Fuzzy System (NFS) approach to perform the instantaneous estimation of the observation noise covariance matrix parameters. Independent NFS are used for each parameter, consisting of thre IF-THEN fuzzy rules defined on the covariances of the innovation sequences, which in turn are estimated by a moving average filter. They perform simulations of SLAM navigation on a 2D simplified synthetic world, where they experiment with induced systematic errors, so their advance to real world conditions is a compelling challenge. In [17] a Takagi-Sugeno approach is applied to approximate the local behavior of the system, then it is decomposed in a set of linear systems that are dealt with by conventional DKF; the output of the decoupled systems is linearly combined to obtain an estimate of the global system state. Again, results are shown on simulation environments and the T-S system parameters must be trained off-line on accurate sampling of the data that the system will encounter. To approach on-line performance some authors built their fuzzy inference system based on theoretical physical understanding of the robot-environment-sensing relations, such as in the work reported in[18] where the GPS measurements noise covariance is estimated by this means.

Another way to mix Fuzzy Systems and EKF is performing the Kalman gain estimation by Fuzzy techniques. This approach is demonstrated in [16] on a prototype land mine detector robot. One critical feature of this robot is an accurate map of the terrain undulations, needed to align the sensor with terrain. The DKF is used to maintain an accurate estimation of the terrain model integrating the laser rangefinder readings. To avoid non-linear modelling, the Kalman gain is set according to the terrain classification performed by a Takagi-Sugeno system, trained on measurement samples from an in-house terrain database.

Finally, a way to improve convergence of the EKF is manipulate the state covariance predictor multiplying it by a diagonal matrix of "fading memory factors", which is equivalent so a low pass FIR applied to the state covariance predictor. A suboptimal algorithm to set the filter coefficients has been called the Strong Tracking Kalman Filter (STKF). In [10] an adaptive method to set the STKF parameters has been proposed, based on a Takagi-Sugeno fuzzy system defined on the innovation (the difference between the predicted and observed sensor information) divergence. However, the rules of the Takagi-Sugeno are built beforehand, with membership functions defined arbitrarily. Results are shown on simulations of GPS based localization of mobile systems.

\subsection{Information fusion}

The state representation and dynamics of the KF type algorithms can be combined with the results of other CI techniques, allowing for the increase in robust- 
ness and a kind of symmetric validation in some cases. EKF has been used along with Fuzzy Occupancy Maps (FMO) to perform localization of legged robots in the context of Robot Soccer [13]. The FMO is a grid representation of the playing field, each cell in the grid has a fuzzy ocuppancy value which is updated when the robot performs a movement. Updating is done by blurring the FMO in the direction of commanded movement, and correcting the position estimate on the basis of visual detection of known landmarks. The state definition of the EKF is the robot position and orientation, and an analytical expression for the Jacobian matrix is given. The fusion of both methods allows the easy initialization of the EKF, which performs a computationally efficient local process of continued localization of the robot, increasing the accuracy of the FMO with large cell sizes. One way to realize the fusion of both methods consists in running the EKF and the FMO in parallel, using each other to confirm the results, being the FMO the main reference. When occupancy fuzzy values are low, the system tends to confuse. Then a population of EKF's are running in parallel, maintaining competing hypothesis about the robot position. The ones that show lower agreement with the FMO can be removed. Another way to perform the representation fusion is reported in [2], where a collection of KF is used with diverse noise covariances, the resulting estimation is fused by a Takagi-Sugeno Fuzzy System, providing an improved traking in mobile localization.

\section{Conclusions}

In summary KF approaches have used as estimation methods for learning processes as well as they have used learning processes to estimate some of their computational elements, namely the noise covariance, the Kalman gain or the non-linear model linear approximation. The $\mathrm{KF}$ aproach provides a convenient way to adapt the estimation process to improve convergence, while, on the other hand, its linear assumptions are too strong for most real-life applications, so that non-linear learning algorithms help to make the KF approaches applicable to applications such mobile robot navigation.

There are a number of approaches that will be worth to explore, like embedding DKF or EKF into Evolutionary Strategies (ES) in order to use the uncertainty management of KF approaches to improve the self-tuning of the ES parameters, or to deal with uncertain fitness functions. Also, there are few works on the non-linear modelling of the sensor information, so that non-linear observation functions could be learned from the data, even on-line. Training Neural Networks or other Computational Intelligence tools for this functionality can be a promising line of research.

One avenue for further research in the application of the KF and hybridized approaches is that of Multi-Robot Systems, such as swarms of modular robots. For instance, it would be appealing to explore the application of distributed $\mathrm{KF}$ approaches to task like the cooperative map building among swarms of autonomous robots [5]. 


\section{References}

1. T. Bailey and H. Durrant-Whyte. Simultaneous localization and mapping (slam): part ii. Robotics \& Automation Magazine, IEEE, 13(3):108-117, 2006.

2. R. Carrasco and Cipriano A.. Fuzzy logic based nonlinear kalman filter applied to mobile robots modelling. In 2004 IEEE international conference on fuzzy systems, volume 3, pages 25-29, 2004.

3. A. Chatterjee and F. Matsuno. A neuro-fuzzy assisted extended kalman filterbased approach for simultaneous localization and mapping (slam) problems. Fuzzy Systems, IEEE Transactions on, 15(5):984-997, 2007.

4. Minyong Choi, R. Sakthivel, and Wan Kyun Chung. Neural network-aided extended kalman filter for slam problem. In Robotics and Automation, 2007 IEEE International Conference on, pages 1686-1690, 2007.

5. M. Di Marco, A. Garulli, A. Giannitrapani, and A. Vicino. Simultaneous localization and map building for a team of cooperating robots: a set membership approach. Robotics and Automation, IEEE Transactions on, 19(2):238-249, Apr 2003.

6. M.W.M.G. Dissanayake, P. Newman, S. Clark, H.F. Durrant-Whyte, and M. Csorba. A solution to the simultaneous localization and map building (slam) problem. Robotics and Automation, IEEE Transactions on, 17(3):229-241, 2001.

7. H. Durrant-Whyte and T. Bailey. Simultaneous localization and mapping: part i. Robotics $\&$ Automation Magazine, IEEE, 13(2):99-110, 2006.

8. Matthias O. Franz and Hanspeter A. Mallot. Biomimetic robot navigation. Robotics and Autonomous Systems, 30(1-2):133-153, January 2000.

9. S. J. Julier and J. K. Uhlmann. A new extension of the kalman filter to nonlinear systems. In Proceedings of AeroSense: The 11th International Symposium on Aerospace/Defence Sensing, Simulation and Controls, 1997.

10. Dah-Jing Jwo and Sheng-Hung Wang. Adaptive fuzzy strong tracking extended kalman filtering for gps navigation. Sensors Journal, IEEE, 7(5):778-789, 2007.

11. R.E. Kalman. A new approach to linear filtering and prediction problems. Transaction of the ASME - Journal of Basic Engineering, March:35-45, 1960.

12. J. Mandel. A brief tutorial on the ensemble kalman filter. Technical Report CCM Report 242, University of Colorado at Denver and Health Sciences Center, February 2007.

13. F. Martin, V. Matellan, P. Barrera, and J.M. Cañas. Localization of legged robots combining a fuzzy-markov method and a population of extended kalman filters. Robotics and Autonomous Systems, 55(12):870-880, December 2007.

14. Qinggang Meng and Mark Lee. Error-driven active learning in growing radial basis function networks for early robot learning. Neurocomputing, 71(7-9):1449-1461, March 2008.

15. K. Murphy and S. Russell. Sequential Monte Carlo Methods in Practice, chapter Rao-Blackwellised particle filtering for dynamic bayesian networks. SpringerVerlag, January 2001.

16. Homayoun Najjaran and Andrew Goldenberg. Real-time motion planning of an autonomous mobile manipulator using a fuzzy adaptive kalman filter. Robotics and Autonomous Systems, 55(2):96-106, February 2007.

17. C.D. Pathiranage, K. Watanabe, and K. Izumi. Simultaneous localization and mapping (slam) based on pseudolinear measurement model with a bias reduction approach. In Industrial and Information Systems, 200\%. ICIIS 200\%. International Conference on, pages 73-78, 2007. 
18. G. Reina, A. Vargas, K. Nagatani, and K. Yoshida. Adaptive kalman filtering for GPS-based mobile robot localization. In Safety, Security and Rescue Robotics, 200\%. SSRR 200\%. IEEE International Workshop on, pages 1-6, 2007.

19. D Simon. Training fuzzy systems with the extended kalman filter. Fuzzy Sets and Systems, 132(2):189-199, 2002.

20. D Simon. Training radial basis neural networks with the extended kalman filter. Neurocomputing, 48:455-475, 2002.

21. R. Smith, M. Self, and P. Cheeseman. Autonomous Robot Vehicles, chapter Estimating uncertain spatial relationships in robotics, pages 167-193. Springer-Verlag, 1990.

22. R. C. Smith and P. Cheeseman. On the representation and estimation of spatial uncertainty. Technical Report TR 4760 \& 7239, SRI, 1985.

23. S. Thrun. Exploring Artificial Intelligence in the New Millenium, chapter Robotic Mapping: A Survey. 2002.

24. G. Welch and G. Bishop. An introduction to the kalman filter. In SIGGRAPH 2001 In Computer Graphics, Annual Conference on Computer Graphics $\&$ Interactive Techniques. ACM Press, 2001. 